\title{
Development of a Self-playing Piano with MIDI File
}

\author{
Chang-Yong Lee ${ }^{1}$, Young-Hyung Kim ${ }^{2}$, Jong-Tae Sung ${ }^{3}$ and Yong-Hwan Lee \\ ${ }^{1}$ First Author, Department of Electronics Engineering, Kumoh National Institute of \\ Technology, Gumi, 730-701, South Korea. \\ ${ }^{2}$ Department of IT Convergence, Kumoh National Institute of Technology, \\ Gumi, 730-701, South Korea. \\ ${ }^{3}$ Dynatone, Gumi, 730-350, South Korea. \\ ${ }^{4}$ Corresponding Author, School of Electronics Engineering, Kumoh National \\ Institute of Technology, Gumi, 730-701, South Korea. \\ E-mail: lcy42845220@gmail.com \\ Abstract
}

\begin{abstract}
This paper presents the development of an automatic piand which plays by itself from MIDI(Musical Instrument Digital Interface) file. We an find the MIDI format file in music data files or play data files. Piano tone is extracted from the MTDI file and the sync time is judged and decided from the extracted piano tone so that the sync time can be sent to master CPU. These data are sent to slave CPU and then to the sound board and solenoid driving circuits. We research and develop sound trace software and sound trace board. This is one of the key technologies for a digital piano keyboard. It transmits the extracted data to the key motion Control device which makes the piano playing automatically. Due to the large sound ata, NAND lash memory is used to handle the data. Because the read/write speed is very important, main memory is required to be designed as a very fast structure. We designed it to fit the length of the PCB pattern address line. Because we expect large electrical current, SMPS(Switching Mode Power Supply) should be designed in low volume and weight with high power efficiency. We add a regulator to the circuit to supply the constant voltage for the circuit to prevent possible damages. Regulators are $5 \mathrm{~V}$ and $24 \mathrm{~V} 5 \mathrm{~V}$ regulator is for $\mathrm{MCU}$ and $24 \mathrm{~V}$ regulator is for solenoid. We designed the optimun key-scan circuit for controlling the 88 keyboards and designed the serial controlfor the reduced number of wires. The self-playing piano has high growth polential in superior educational effectiveness and a variety of usability. This technology is also likely to be applied to a medical device as music therapy.
\end{abstract}

Keywords: Sound trace board, MIDI, Sync Time, PCB, Solenoid

\section{Introduction}

Digital player piano is self-playing piano in accordance with tone signals. Commonly, the digital player piano consist of music file entry system, music file output system and piano module. The piano module controls the movement of the keyboards. The digital player piano uses solenoids and music files. The solenoids are in the piano module. We can find the necessary data in the music file.

Core technology of the digital player piano is to implement the auto-play function. In detail, the operation of the 88 keyboards corresponds to the multi-instrument voices to be input to the instrument. It needs a technique to control simultaneous, sequential and continuous control. The sound source matches the multi-keyboard (operating time, speed, intensity) for quick and accurate outputs.

Sound tracking technology finds the piano tones. The source tracking technology uses a variety of instruments (GM 128 different instrument) but finds only the piano tones in 
music production file. It tracks the piano tones while playing the music file. The piano tones note number, velocity values, note on time and note off time are in the music file. Trace board uses high-performance (32bit) microprocessor to apply tracking algorithm.

We develop driving circuit for the accurate and reliable operation of the variable speed electronic solenoid and power supply circuit. We design the filter circuit for driving the solenoid, the optimum key-scan circuit for controlling the 88 keyboards, the serial control for reduced wires and control circuit of response speed with less than $100 \mathrm{~ms}$.

\section{Development of Piano Sound Trace Board}

Trace board analyzes music files. In the music file, the trace board looks for MIDI file to extract the tone of piano. That determines sync time from piano tones. While tracking the sync time to synchronize with the beat of the music, the trace board sends note number, velocity value, the length of the sound and note on/off time to key motion controller and sound chip. Figure 1 illustrates the flowchart of trace board function.

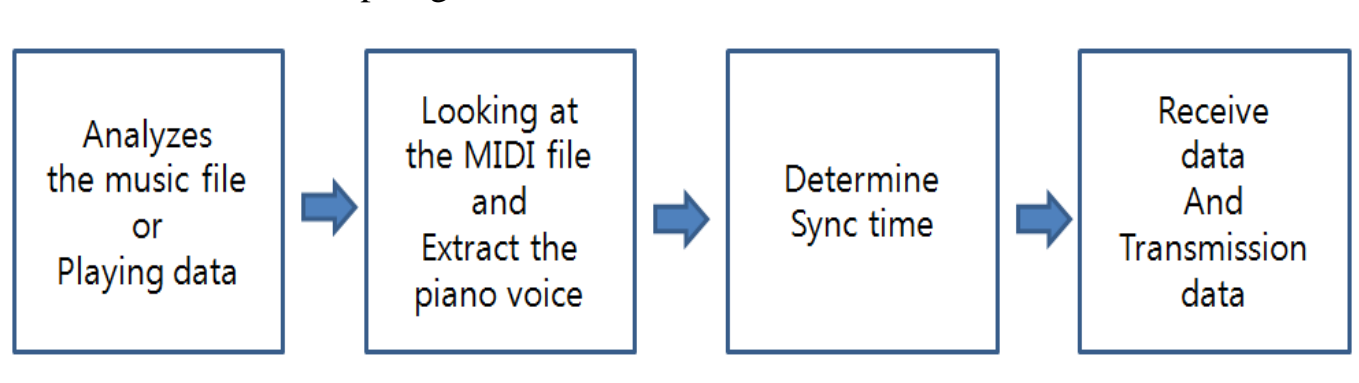

\section{Figure 1. Flowchant of Trace Board Function}

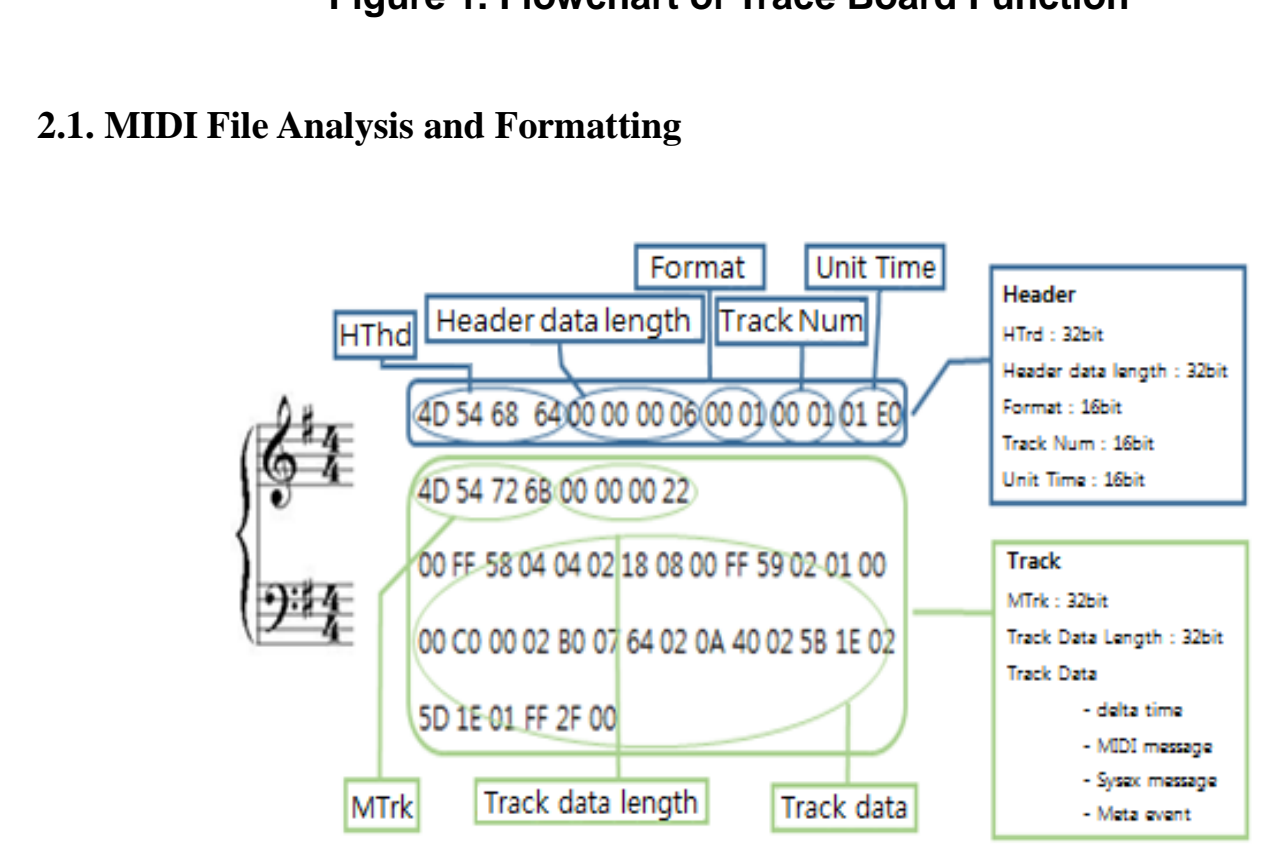

Figure 2. MIDI File Format

MIDI (Musical Instrument Digital Interface) is a technical standard that describes a synthesizer, sequencer, computer and connectors and allows a wide variety of electronic musical instruments, computers and other related devices to connect and communicate with one another. MIDI is a sound source input device for converting a digital signal, so that the music instruments can be accessed in digital communication. It provides compatibility with the input and output of the music instruments to handle between the 
instrument and the instrument or between instruments and computers to digital signals. It is a standard specification for hardware and data structures. MIDI file consist of a header part and track part. The header section of the MIDI file includes $\mathrm{HT}_{\mathrm{hd}}$, header data length, track number, unit time and format files. The track section of the MIDI file includes $\mathrm{MT}_{\mathrm{rk}}$, track data length and track data, as illustrated in Figure 2. The track data are real music data which includes delta time, MIDI messages, sync messages and meta messages. The track data is analyzed by piano sound trace software.

\subsection{Piano Sound Extraction Algorithm Design and Sync Time Judgment}

We develop software for MIDI file to extract the tone of piano and keyboards motion. The developed algorithms are for sound synchronization. Trace board receives sound data from the sound board. The trace board analyzes the MIDI file in the received data. The board can extract the tone of piano from MIDI file. The sync time judges and determines from the tone of piano. We design the $\mathrm{H} / \mathrm{W}$ and $\mathrm{S} / \mathrm{W}$. Running data of keyborrd are transmitted to the key motion controller, as illustrated in Figure 3.

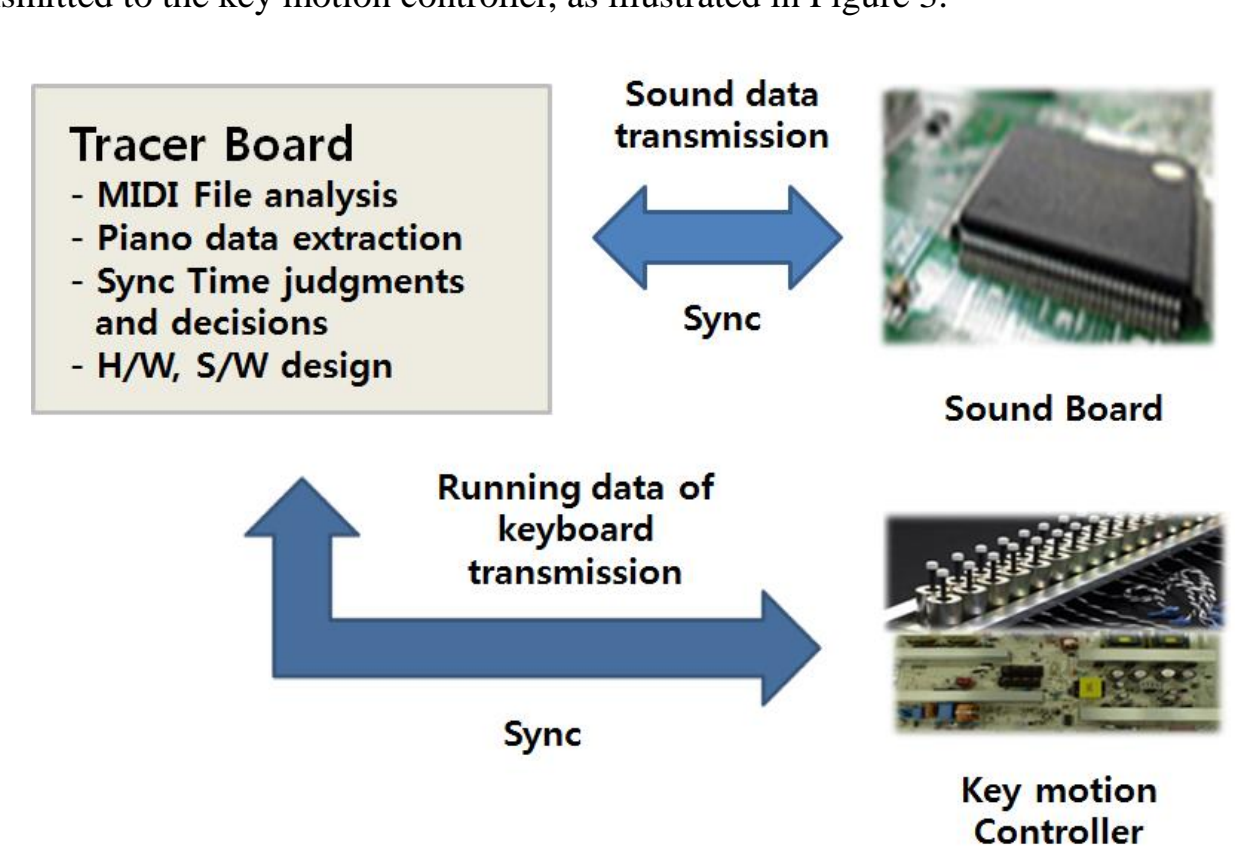

\section{Eigure 3. Software Algorithm Overview}

An acoustic piano needs to tune the strings by expert periodically. Tuning for conventiona digital piano is not easy even if velocity value is changed due to contact resistance change or a foreign object of the sensor keyboard. The self-tuning keyboard function is implemented in our digital piano. User will read the resistance value of the physical keyboard sensor by using the buttons. The self-tuning keyboard function is automatically available after calculating the contact resistance compared to the default value of the initial production.

Figure 4 illustrates the flowchart of mode selection with tuning mode. If the digital piano starts, we can select a mode by using a hidden key. Input of the hidden key execute tuning mode. The tuning mode starts with the keyboard scan and the keyboard input check. Depending on the check status, another scan is performed or passed to the next steps. If the tuning mode is finished or the hidden key is not entered, the digital piano will execute playing the piano mode. 


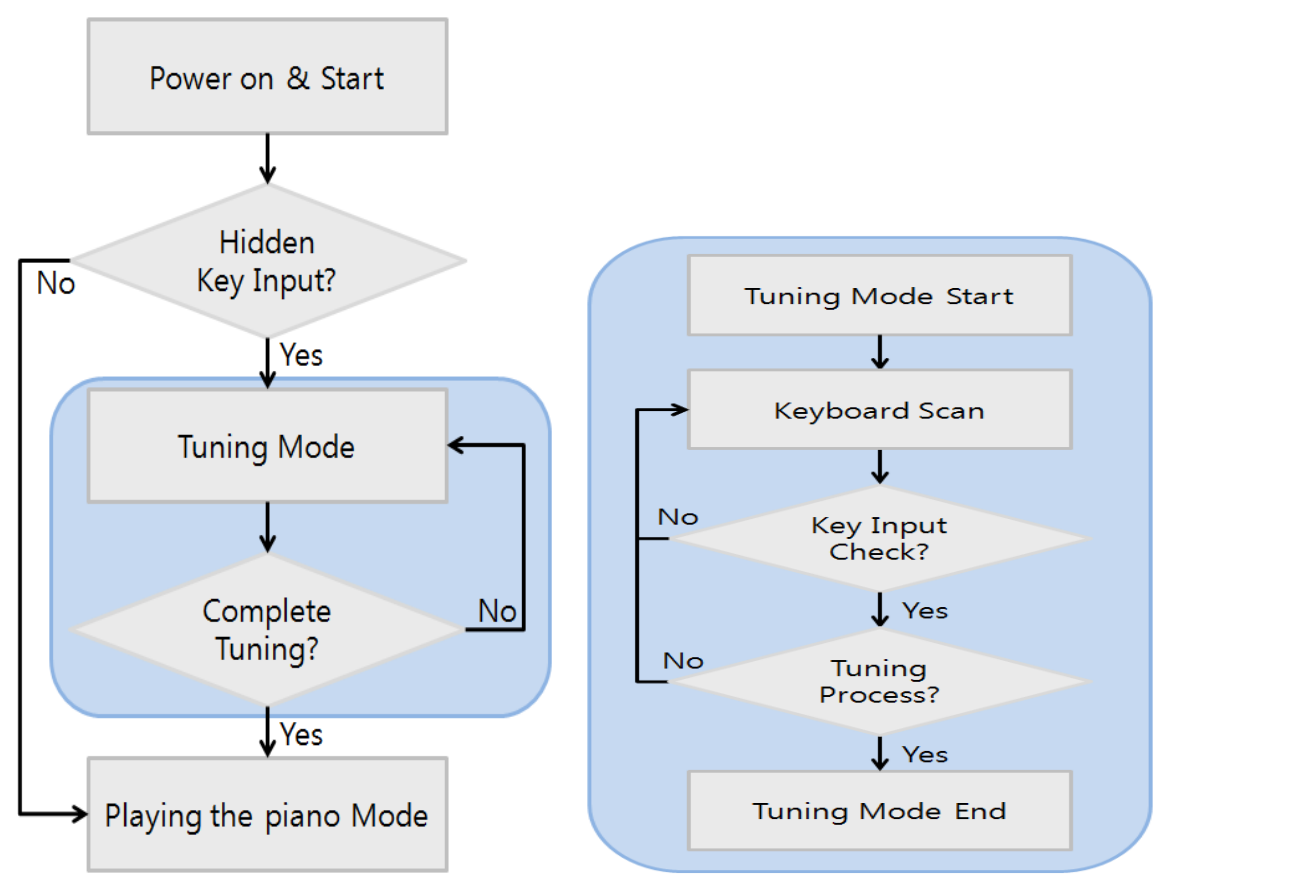

Figure 4. Flowchart of Mode Selection with Tuning Mode

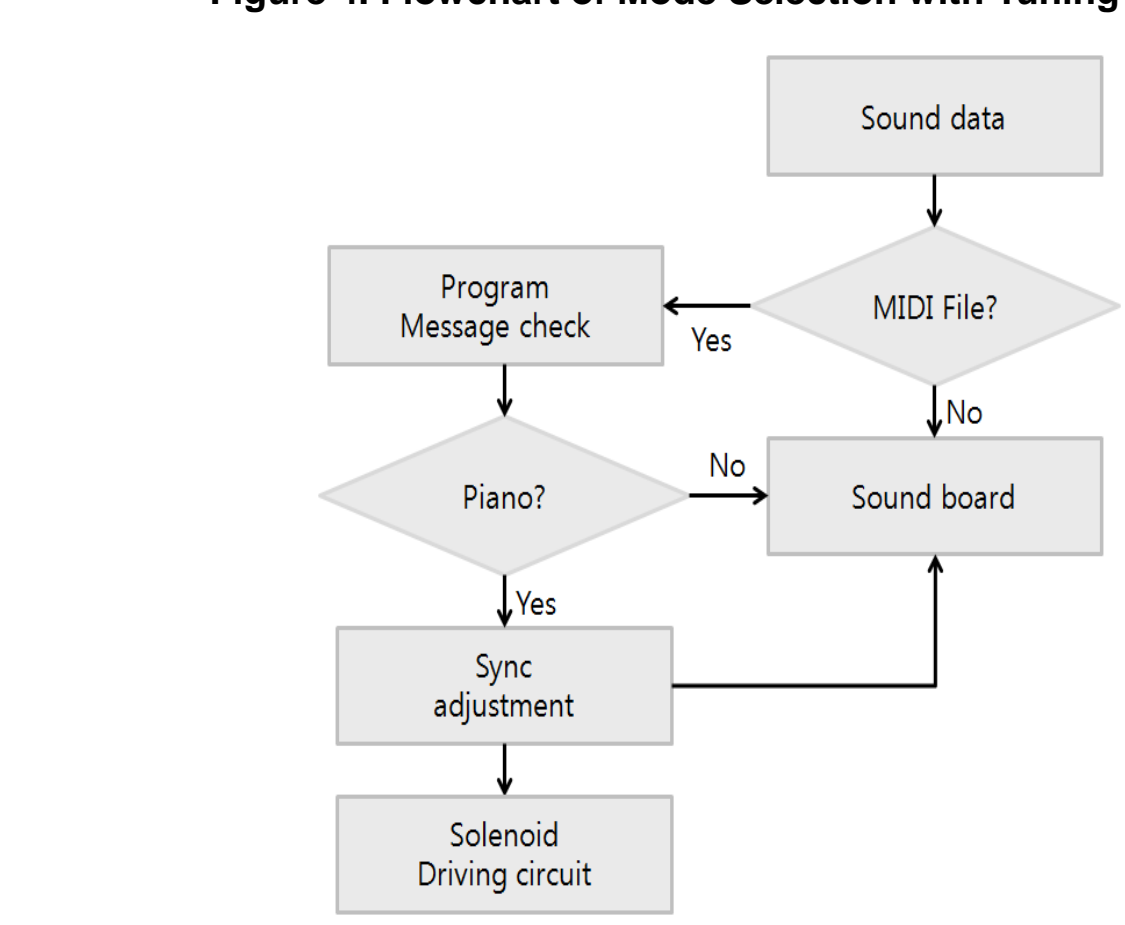

Figure 5. Flowchart of Sync Time

Figure 5 illustrates the flowchart of sync time. We check MIDI file from the sound data. If there are not MIDI files in the sound data, the files will be sent back to sound board. The program message is displayed when sound data has MIDI files. Trace board extracts the tones of piano from MIDI file. If MIDI file has not tone of piano, it will be sent to sound board. Sync time is defined as the tone of piano. The sync time is able to send from the tracer board to the sound board and solenoid driving circuits. 


\subsection{Tracer Board Circuit Design (H/W)}

NAND flash memory is used to store the data, because the read/write speed is very important, main memory is designed as a fast structure. Data line (16Bit) is considered to be designed in the same direction and the same length. We avoid as much as possible via in the design. We design a GND layer and a separate VCC layer structure with four or more layers. We use to different width of the ground plane and power plane. We will illustrate PCB pattern, design drawing of tracer board circuit and block diagram of tracer board circuit in Figure 6-9.

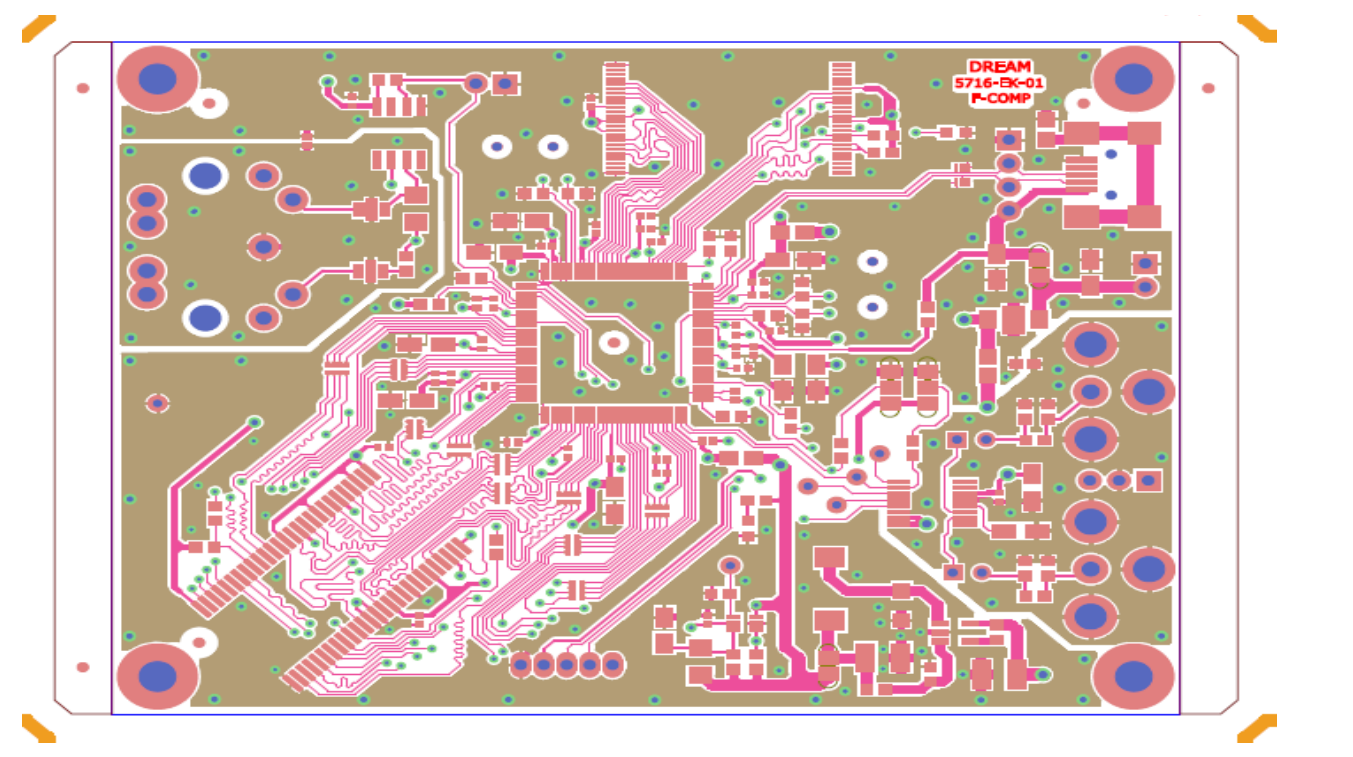

Figure 6. PCB Pattern Reference Drawing by Sound Chip Dream Company

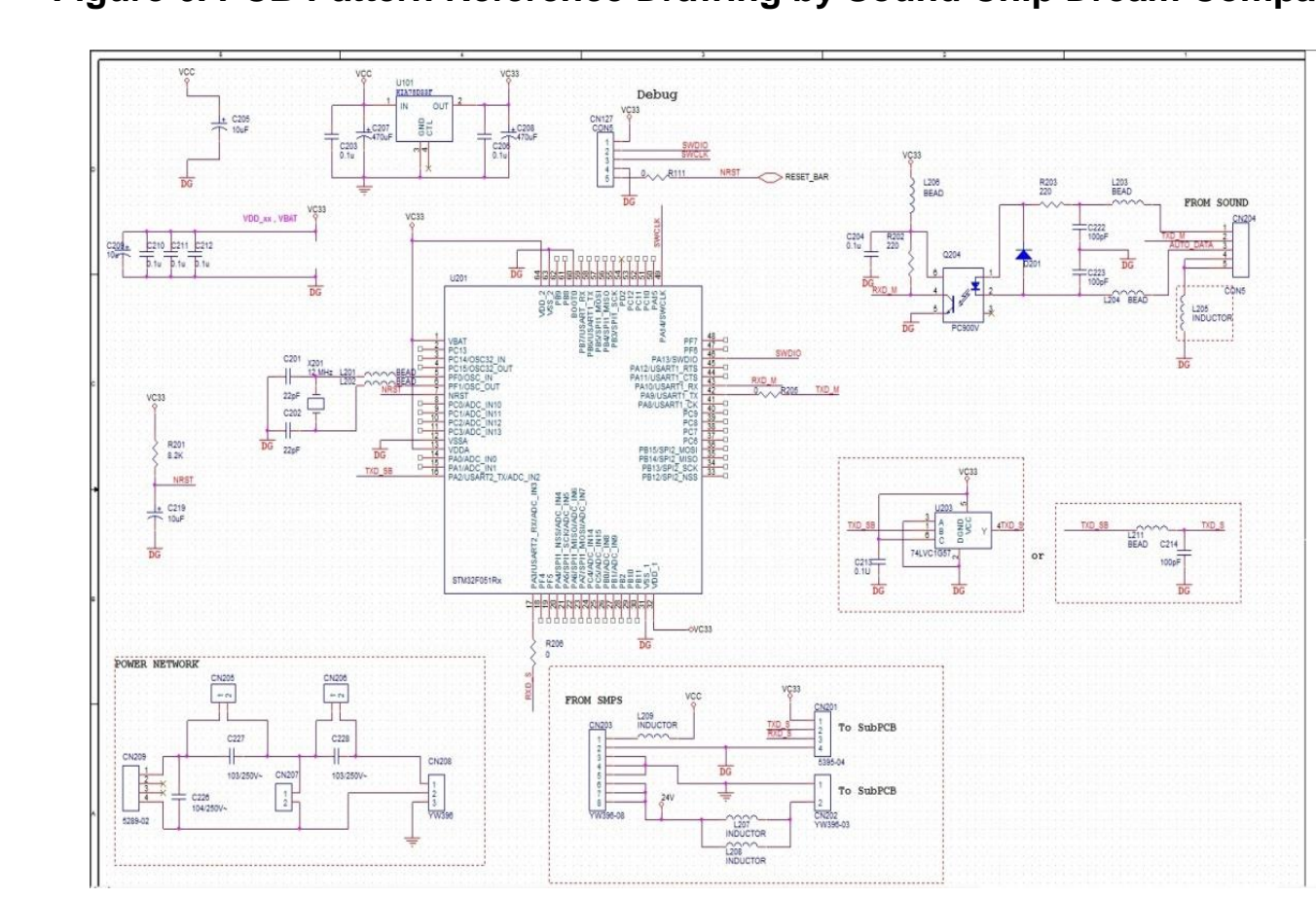

Figure 7. Design Drawing of Tracer Board Circuit (Master) 


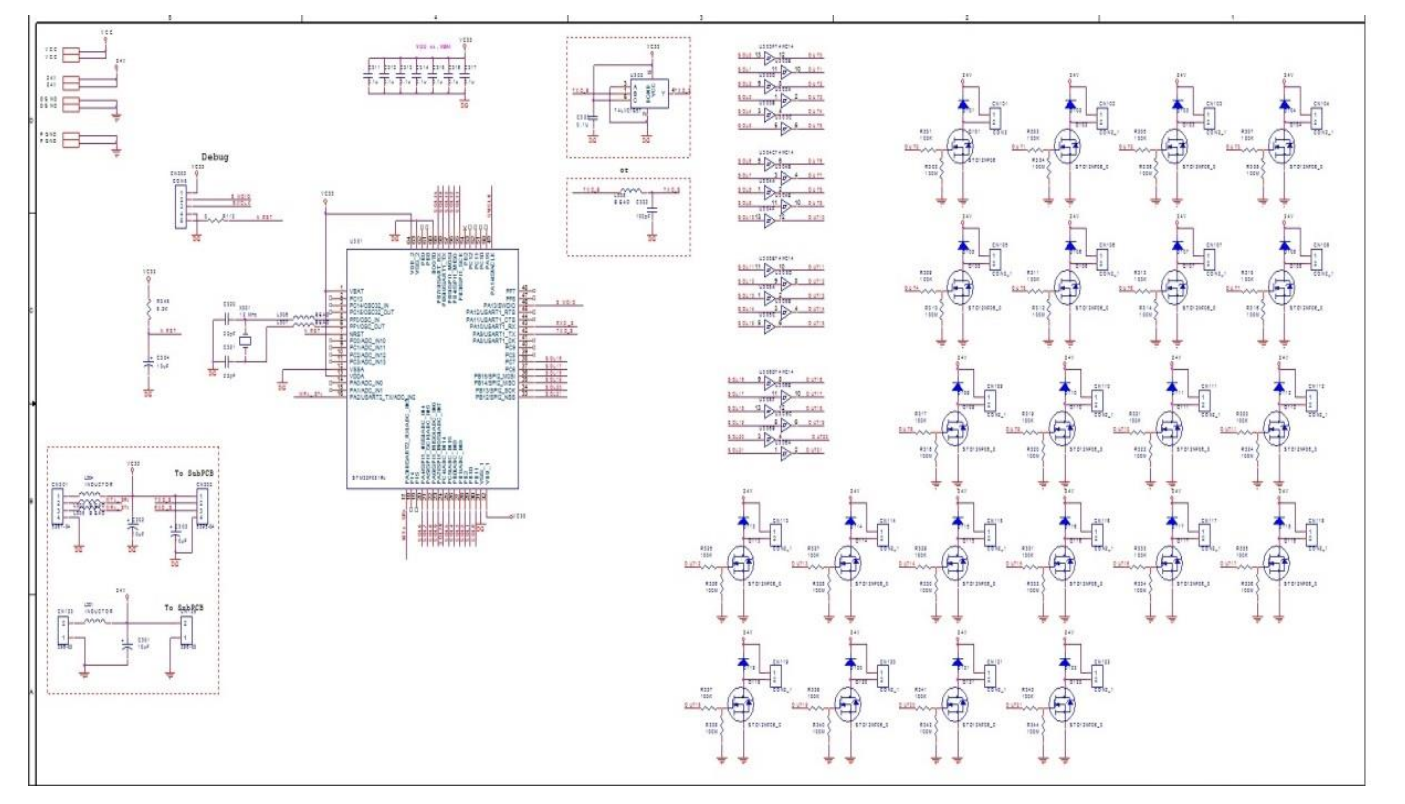

Figure 8. Design Drawing of Tracer Board Circuit (Slave)

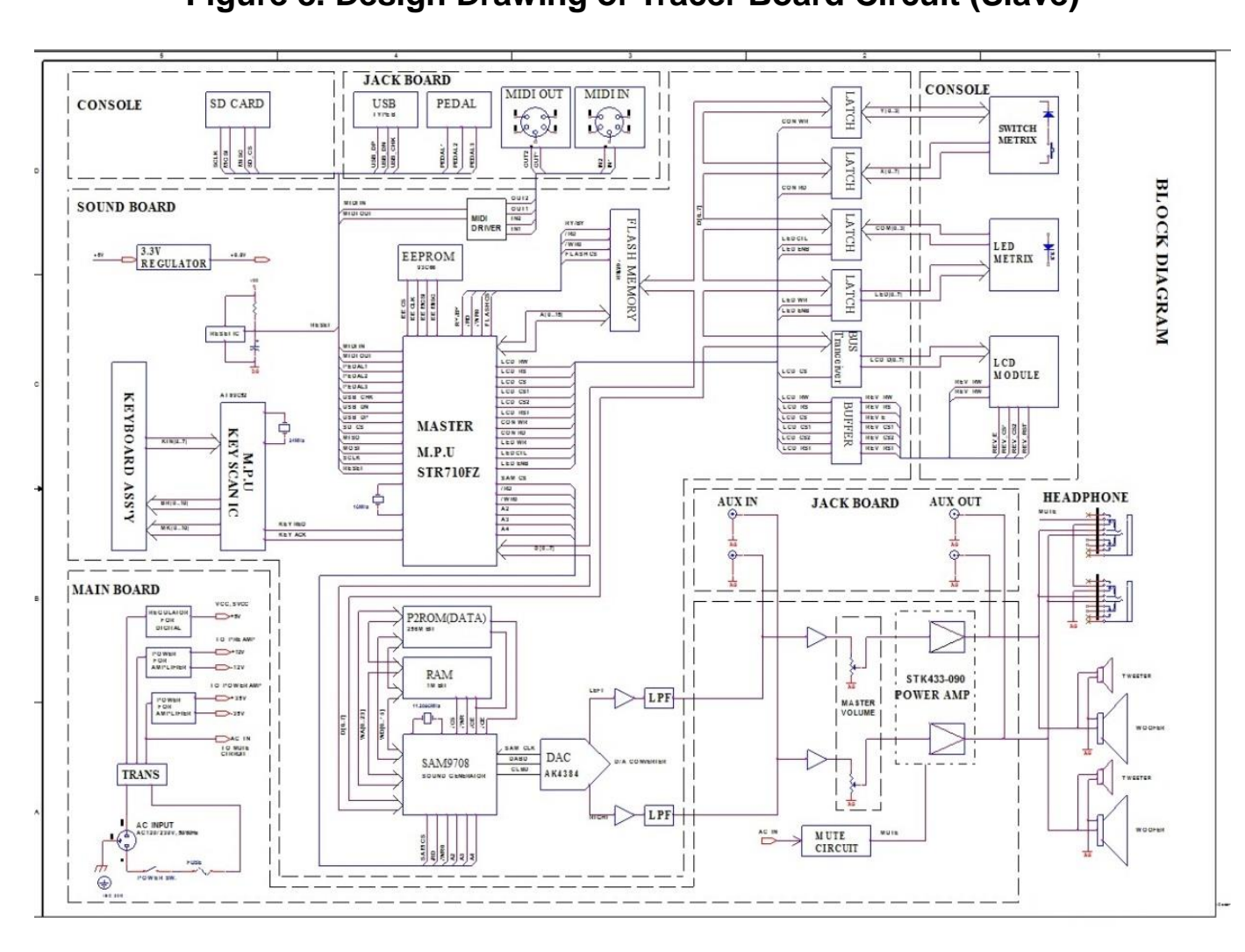

Figure 9. Block Diagram of Tracer Board

\section{Development of Solenoid Driving Circuit}

We develop driving circuit for the accurate and reliable operation of the variable speed electronic solenoid. We also develop power supply circuit. A separate power supply is designed for a stable power supply. SMPS is designed in low volume and weight with high power efficiency. We add a regulator to the circuit to supply the constant voltage for 
the circuit to prevent possible damages. Regulators are $5 \mathrm{~V}$ and $24 \mathrm{~V}$. $5 \mathrm{~V}$ regulator is for MCU and 24V regulator is for solenoid. We design a multi-output circuit for reduce noise due to the voltage difference between the control unit and the drive unit. Block diagram, schematic and design criteria are shown in Figure 10-12 and Table 1.

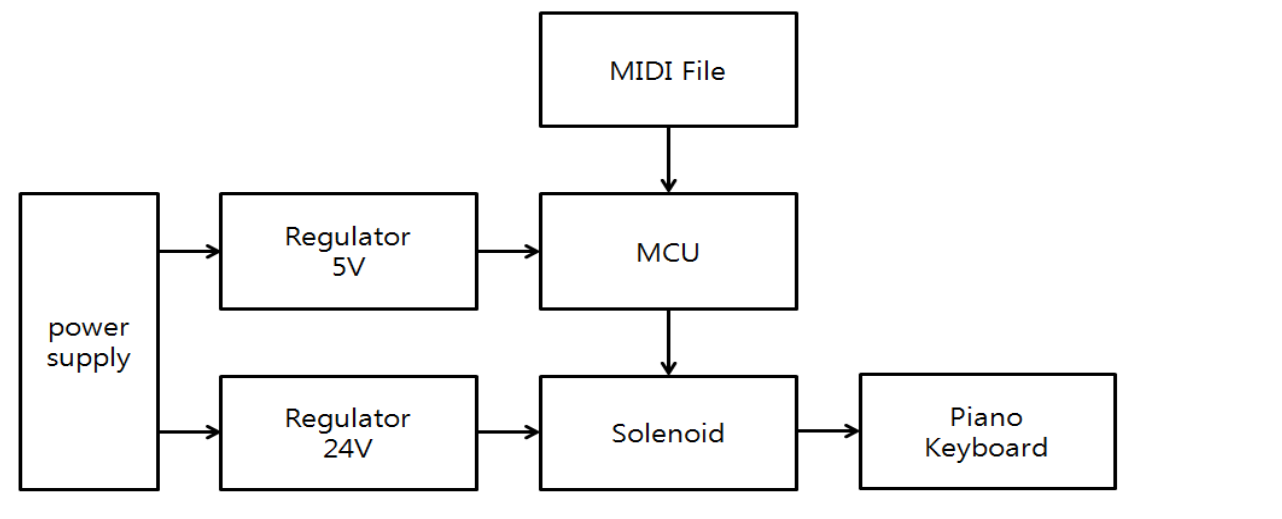

Figure 10. Driving Circuit Block Diagram of the Solenoid

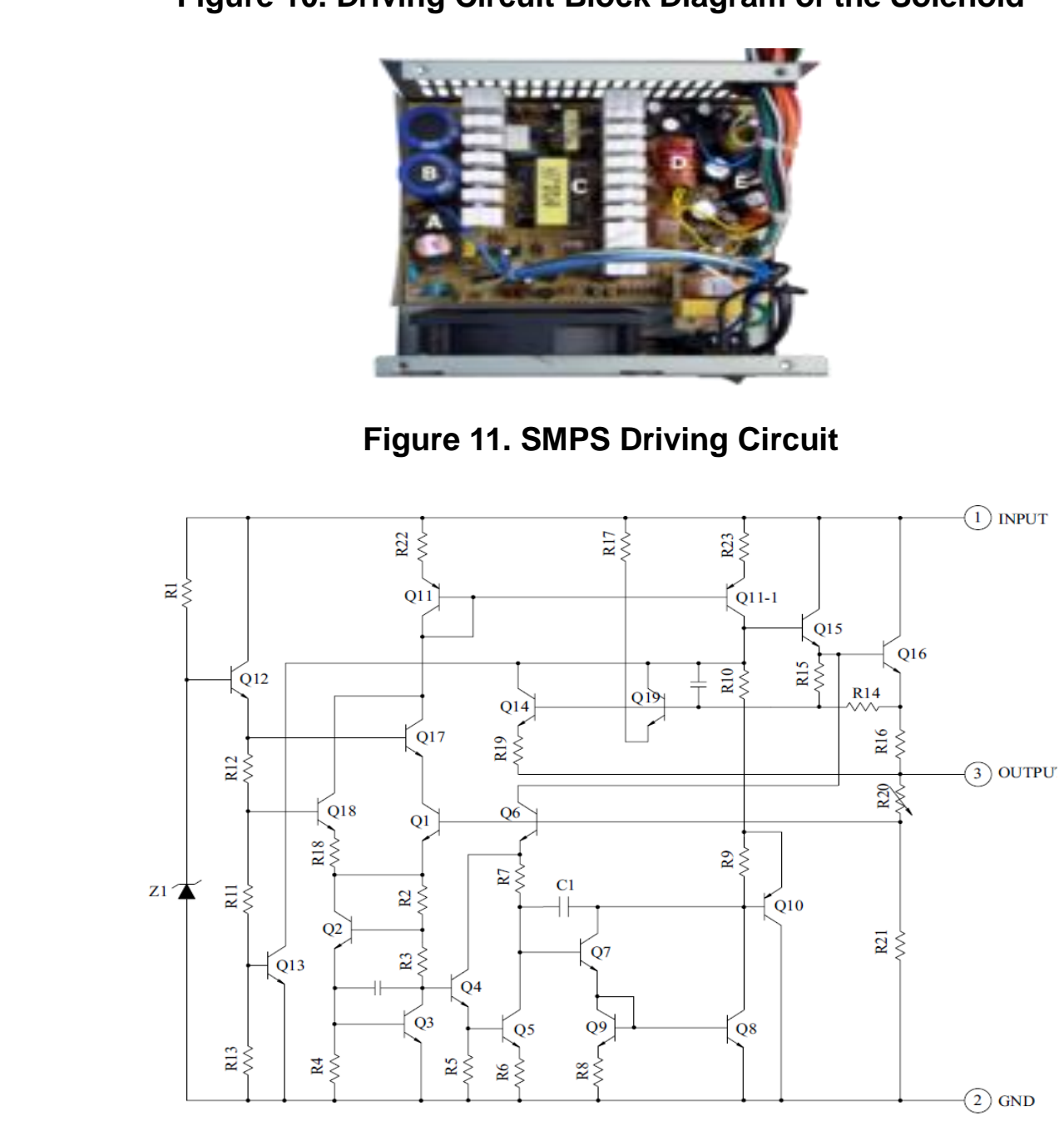

Figure 12. Solenoid Circuit 
Table 1. Design Criteria

\begin{tabular}{|c|c|}
\hline & Baseline \\
\hline Maximum Output Voltage & $5.3 \mathrm{~V}$ or less \\
\hline Minimum Output Voltage & $4.7 \mathrm{~V}$ or over \\
\hline Maximum Operating Temperature & $50^{\circ} \mathrm{C}$ or less \\
\hline Minimum Operating Temperature & $-10^{\circ} \mathrm{C}$ or over \\
\hline
\end{tabular}

We design the filter circuit for driving the solenoid, the optimum key-scan circuit for controlling the 88 keyboards and designed the serial control for reduce wires. We design it considering the high current of PCB pattern. The selection of the MOSFET is for switching solenoids and MCU selection to control signals. We design control circuit of response speed with less than 100ms, as illustrated in Figure 13-14.

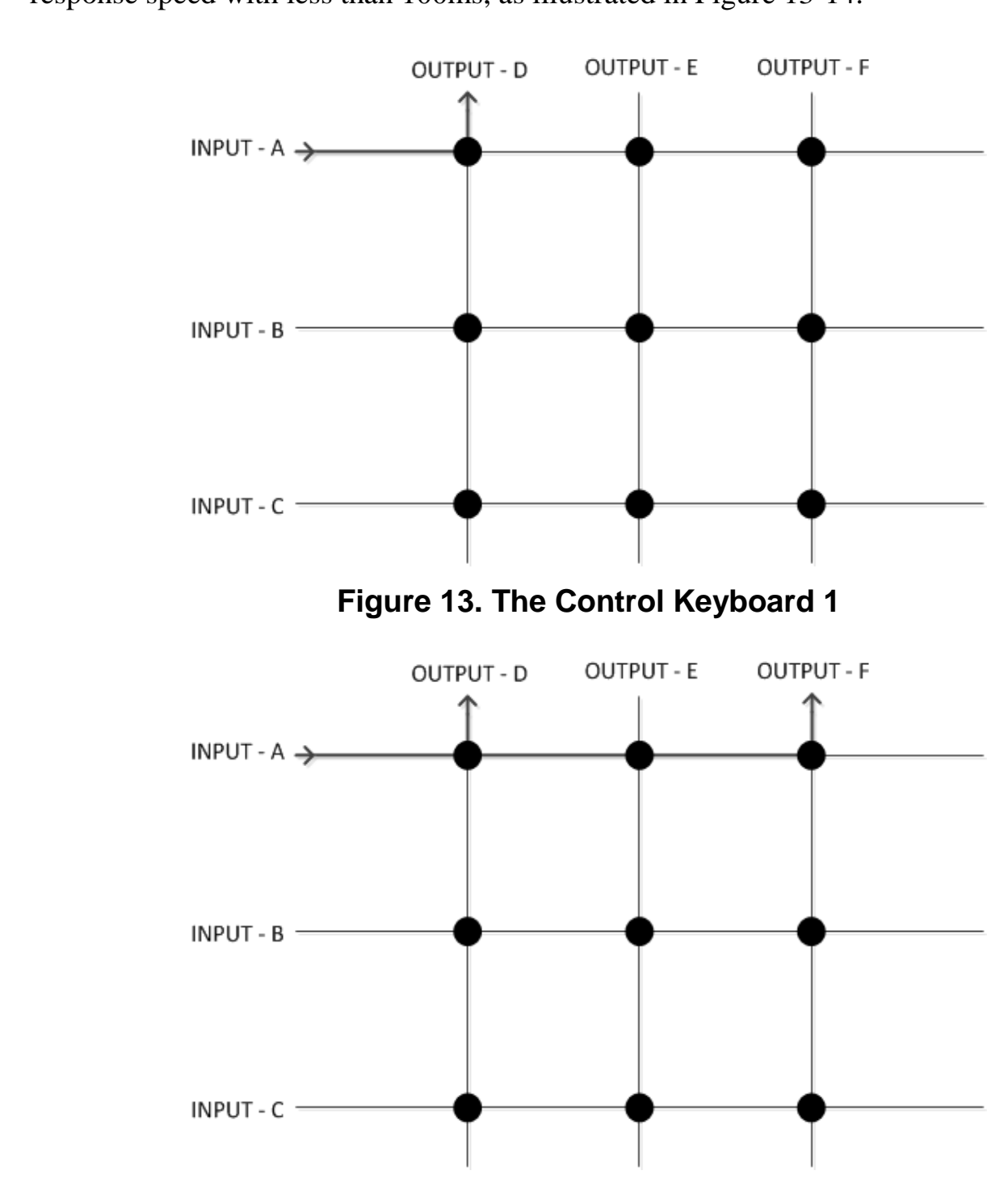

Figure 14. The Control Keyboard 2

\section{Applications}


If the auto-play function implemented in the digital piano, it will have high growth potential in superior educational effectiveness and a variety of usability. The music academy or school also can use this piano mainly for educational purposes. There also are cases purchasing an electronic piano for entertainment as well as education.

This technology can be applied to many musical instruments such as synthesizers, MIDI keyboards, guitars and drums. Users can leverage the interlocking of digital pianos and smart terminal. Users are capable of high-level composition without professional composer equipment. Users can more easily play to use the smart terminal. Conventional player piano was designed to play an expensive acoustic piano. The piano is a large, heavy and instrumental library is expensive. This digital automatic player module complements these disadvantages. We can apply the module to a conventional piano. This technology is also likely applied to a medical device for music therapy. The patients can choice to the music. Patients enjoy the treatment with the help of the robot because, patients can listen to music therapy and play the digital piano.

This technology is capable of spreading to the entire electronic musical instrument industry and another industry.

\section{Conclusion}

We design the S/W and H/W. Trace board receives sound data from the sound board. The trace board analyzes the MIDI file in the received data. The board can extract the tone of piano from MIDI file. The sync timejudges and determines from the tone of piano. Running data of keyboard are transmitted to the key motion controller. The digital piano has tuning mode and playing pianómode. If the digitat piano starts, we can select a mode by using a hidden key. We de elop driving ciculit for the accurate and reliable operation of the variable speed electronie solenoid and SMPS. SMPS is designed in low volume and weight with high power efficiency. We used two regulators to the circuit to supply the constant voltage for the circuit to preyent possible damages. $5 \mathrm{~V}$ regulator is for MCU and 24V regulator is for solenoid. We design it considering the high current of PCB pattern and control circuil of response speed with less than 100ms. The self-playing piano has high growth potential in superior educational effectiveness and a variety of usability. This technology is also likely applied to a medical device for music therapy.

\section{Acknowledgment}

This work is the result of the Business of R\&BD Support for Corporation Promotion funded by Korea Jadustrial Complex Corporation in 2015.

\section{References}

[1] K. Lim and J. C. Lee, "An Electronic Keyboard Instrument Using PC MIDI and USB Interface", ournal of the Korea Society of Computer and Information, vol. 16, no. 11, (2011), pp. 85-93.

[2] H. Zhigang and Z. Changle, "Sci-MIDI toolbox for music research", 2009 IEEE International Workshop, (2009), pp. 159-162.

[3] J. H. Lee, S. Kim and Y. I. Yoon, "Real-time Orchestra Method using MIDI Files", The Journal of the Korea Contents Association, vol. 10, no. 4, (2010), pp. 91-95.

[4] M. R. Kim, "The Research of the Human Computer Interface using by Music XML", Proceeding of KIEE Summer Conference, (2001), pp. 2804-2806.

[5] A. S. Sedra and K. C. Smith, "Microelectronic Circuits", 5th ed., Oxford University, (1997).

[6] H. H. William and Buck, "Engineering Electromagnetics", 7th ed., Mcgraw-Hill, (2005).

[7] S. S. Kim and S. U. Jung, "Hardware Implementation of a Neural Network Controller with an MCU and an FPGA for Nonlinear Systems", International Journal of Control Automation and System, vol. 4, no. 5, (2006), pp. 567-574.

[8] S. I. Do, "Development of self-play system of Digital Piano", 1st ed., Kumoh National Institute of Technology, (2011). 
[9] R. Guevara and G. Wakefield, "A Modal Distribution Approach to Piano Analysis and Synthesis", Proceedings of the International Computer Music Conference, (1996), pp. 350-351.

\section{Authors}

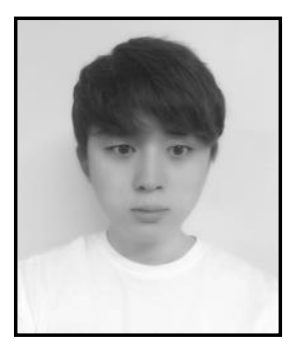

Chang-Yong Lee, $\mathrm{He}$ is studying in electronical engineering in Kumoh National Institute of Technology, Interesting field: SoC, Interface, Embedded system

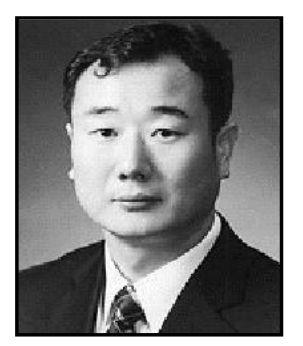

Yong- Hyung Kim, Received the $\mathrm{PhD}$ degree in business administration from the Kumoh National Institute of Technology, Korea, in 2010. He is working as a professor of department of IT convergence at the Kumoh National Institute of Technology. Interesting field : electrical and electronic industrial systems
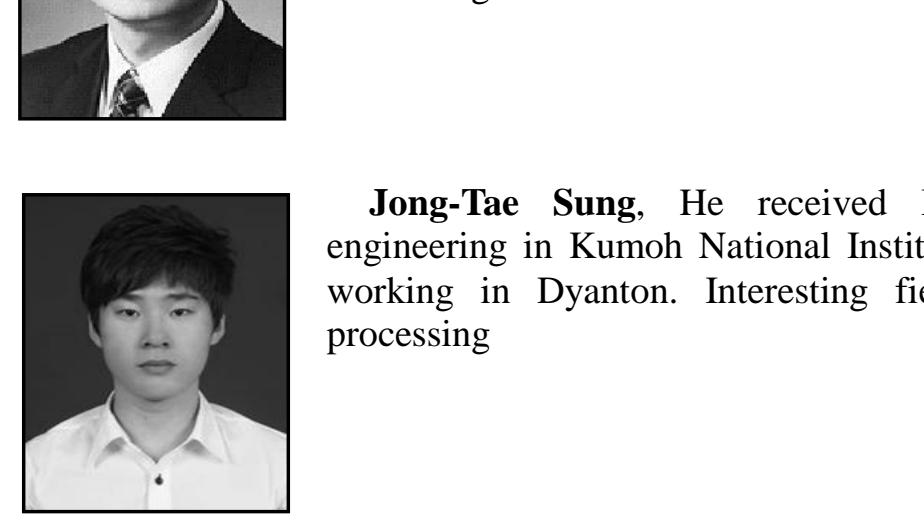

Jong-Tae Sung, He received M.S degree in electronical engineering in Kumoh National Institute of Technology. And he is working in Dyânton. Interesting field : SoC, Interface, Image processing

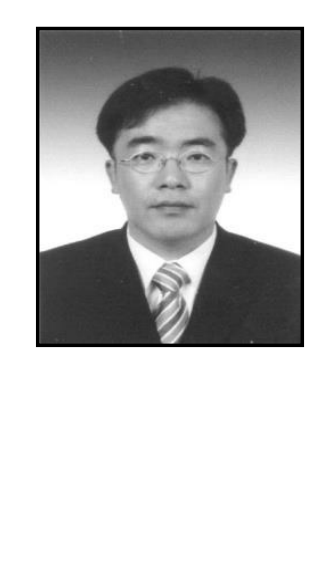

Yong-Hwan Lee, Received the $\mathrm{PhD}$ degree in electronic engineering from the Yonsei University, Korea, in 1999. He is working as a professor of school of electronic engineering at the Kumol National Institute of Technology Interesting field: SoC, Interface, Embedded system, Structure of microprocessor. 